\title{
EL EFECTO PLACEBO EN LOS ENSAYOS CLÍNICOS CON ANTIDEPRESIVOS
}

\author{
Hernán Silva Ibarra*
}

\begin{abstract}
Resumen: Estudios recientes concluyeron que los antidepresivos de nueva generación son ineficaces para tratar las depresiones moderadas o severas. Estadísticamente, no sería muy diferente la mejoría que experimentan estos cuadros con medicamentos o con placebo. Sólo en los pacientes con depresiones más extremas la eficacia de los antidepresivos parece ser significativa. No obstante, la aparente eficacia en este grupo no se debería a la efectividad del medicamento, sino a una menor respuesta al placebo.

Otro grupo de investigadores, empleando una metodología muy similar, llegaron a conclusiones muy diferentes. Los autores sostienen que todos los antidepresivos fueron superiores al placebo, aunque su eficacia real es menor si también se incluyen en el análisis los estudios no publicados.

Esta situación pone de manifiesto las dificultades que enfrenta la investigación de psicofármacos en la depresión y reaviva la polémica respecto de la utilidad y justificación ética del empleo de placebo en esos estudios.
\end{abstract}

Palabras clave: depresión, antidepresivo, placebo

\section{THE PLACEBO EFFECT IN CLINICAL ESSAYS WITH ANTIDEPRESSIVES}

\begin{abstract}
Recent studies determined that antidepressives of new generation were inadequate for moderate or severe depressions. The improvement these diseases show with medication or with a placebo isn't very different, according to statistics. Antidepressives seem to be efficient only upon patients that present an extreme depression. Nevertheless, the apparent efficiency upon this group wouldn't be due to the medication itself, but to a minor response to the placebo. Another group of researchers came to very different conclusions employing a similar methodology. They assured that all antidepressives were superior compared to the placebo, although their real effect would be even minor if analysis of studies not yet published were included.

This situation shows the difficulties the investigation on psychoremedies for depressions present and it revives the polemics about the utility and the ethical justification of employing placebos in this type of studies.
\end{abstract}

Key words: depression, antidepressives, placebo

\section{O EFEITO PLACEBO NOS ENSAIOS CLÍNICOS COM ANTIDEPRESSIVOS}

Resumo: Estudos recentes concluíram que os antidepressivos da nova geração são ineficazes para tratar as depressões moderadas ou severas. Estatisticamente, não seria muito diferente a melhoria que experimentam estes quadros com medicamentos ou com placebo. Somente nos pacientes com depressôes mais extremas a eficácia dos antidepressivos parece ser significativa. Náo obstante, a aparente eficácia neste grupo não seria devido à efetividade do medicamento, senão a uma menor resposta ao placebo.

Outro grupo de pesquisadores, empregando uma metodologia muito similar, chegou a conclusôes muito diferentes. Os autores sustentam que todos os antidepressivos foram superiores ao placebo, ainda que sua eficácia real seja menor se também foram incluídos na análise os estudos não publicados.

Esta situação póe de manifesto as dificuldades que enfrenta a pesquisa com psicofármacos na depressão e reaviva a polêmica a respeito da utilidade e justificativa ética do emprego de placebo nesses estudos.

Palavras-chave: depressão, antidepressivo, placebo

* Clínica Psiquiátrica Universitaria, Hospital Clínico, Universidad de Chile. Chile

Correspondencia: hsilva@med.uchile.cl 
Un estudio publicado recientemente -efectuado por investigadores de universidades británicas, canadienses y estadounidenses- concluyó que los antidepresivos de nueva generación son ineficaces para tratar las depresiones moderadas o severas(1). Estadísticamente, no sería muy diferente la mejoría que experimentan estos cuadros con medicamentos o con placebo. Sólo en los pacientes con depresiones más extremas la eficacia de los antidepresivos parece ser significativa. No obstante, la aparente eficacia en este grupo no se debería a la efectividad del medicamento, sino a una menor respuesta al placebo. El estudio que comentamos se basó en los ensayos clínicos presentados como evidencia a la Food and Drug Administration (FDA) para la aprobación de antidepresivos de nueva generación. Los autores efectuaron un metaanálisis de 37 estudios que incluían 5.133 pacientes, analizaron tanto estudios publicados como no publicados, todos ellos de doble ciego controlados con placebo, en pacientes con trastorno depresivo unipolar, acorde con los criterios del "Diagnostic and Statistical Manual of Mental Disorders (DSM)". Concluyeron que la evidencia para prescribir antidepresivos es escasa y sólo debieran indicarse en pacientes que sufren cuadros depresivos más severos o cuando hayan fracasado los tratamientos alternativos.

Como cabía esperar, estas afirmaciones generaron una aguda polémica en los medios de comunicación y en la comunidad psiquiátrica internacional.

Otro grupo de investigadores, empleando una metodología muy similar -en este caso se trató de un metaanálisis de 74 trabajos con nuevos antidepresivos presentados a la FDA y que incluían a 12.564 pacientes-, llegaron a conclusiones muy diferentes(2). Este grupo sostiene que todos los antidepresivos fueron superiores al placebo, aunque su eficacia real es menor si también se incluyen en el análisis los estudios no publicados.

Conclusiones tan diversas surgen del modo en que los investigadores de ambos grupos interpretaron la relevancia clínica de las diferencias entre la respuesta a los fármacos y al placebo, las que fueron muy similares en los dos estudios.

Esta situación pone de manifiesto las dificultades que enfrenta la investigación de psicofármacos en la depresión y reaviva la polémica respecto de la utilidad y justificación ética del empleo de placebo en esos estudios.

\section{Estudios con placebo en depresión}

Se define como "placebo" a sustancias inertes o a procedimientos de apariencia idéntica al tratamiento farmacológico activo o al tratamiento médico que se está investigando. También se pueden emplear como placebo sustancias activas que producen efectos similares al fármaco en estudio, pero que carecen de la propiedad que se supone terapéuticamente eficaz. Los placebos se emplean con la finalidad de controlar los efectos psicológicos del tratamiento y los resultados que producen se denominan "efecto placebo". En cambio, se denomina "efecto nocebo" a las reacciones adversas a aspectos incidentales del tratamiento; son muy comunes en pacientes y voluntarios sanos que participan en estudios clínicos y una causa común de incumplimiento de los tratamientos(3).

En los pacientes con depresión, la respuesta al placebo parece ser extremadamente alta, lo que arroja dudas sobre la verdadera eficacia de los antidepresivos. Se ha planteado que esto puede deberse a un artificio de los métodos de metaanálisis, ya que los estudios difieren en las estrategias de investigación y en el análisis de los datos. No obstante, cuando otras patologías son sometidas al mismo procedimiento, las cifras de respuesta al placebo son menores. Por ejemplo, un metaanálisis del Quality Assurance Project encontró que el placebo producía un 60\% de la mejoría en la depresión, en cambio sólo era de un $53 \%$ en el trastorno de ansiedad generalizada, $23 \%$ en agorafobia, $21 \%$ en trastorno obsesivo compulsivo y prácticamente $0 \%$ en esquizofrenia(4). Por lo tanto, la elevada respuesta al placebo en los estudios sobre depresión parece obedecer a otra causa.

Diferentes factores pueden contribuir a la elevada respuesta a placebo en los estudios con antidepresivos. Entre ellos se encuentran las características de las enfermedades crónicas, el fenómeno estadístico de regresión a la media, el curso natural de la depresión, las limitaciones de las escalas para medir los síntomas depresivos y el efecto beneficioso de estar en tratamiento.

Las personas con enfermedades crónicas, como la depresión, generalmente buscan ayuda cuando se agravan sus síntomas. Por las fluctuaciones naturales del cuadro es probable que hayan mejorado en la siguiente evaluación. Los sucesos vitales influyen en la depresión: los negativos la empeoran y los positivos la mejoran. Las situaciones adversas a veces se resuelven por sí mismas. Experiencias positivas inesperadas pueden iniciar un 
proceso de mejoría de los síntomas depresivos. Cambios en el soporte social de los pacientes también pueden influir en los síntomas.

La regresión a la media en medidas sucesivas es un fenómeno puramente estadístico que ocurre cuando los participantes son elegidos por tener temporalmente elevadas puntuaciones en un instrumento de evaluación. Si los individuos se encuentran cerca del límite superior teórico de la escala, en las mediciones siguientes tendrán puntuaciones más moderadas, independientemente de si hayan recibido alguna intervención. Por lo tanto, se trata de un fenómeno estadístico aleatorio, independiente de los efectos del fármaco y que afecta tanto al grupo en estudio como al grupo control.

Por otra parte, la depresión es una enfermedad de curso fluctuante y puede presentar remisiones espontáneas. $\mathrm{Al}$ estudiar grandes poblaciones de pacientes se ha encontrado que un episodio depresivo mejora en un promedio de seis semanas y que a las 12 semanas se ha recuperado el $75 \%$ de los casos(5). Supongamos que el tiempo promedio de mejoría fuera de ocho semanas y el $75 \%$ de los pacientes se recuperara a las 16 . En ese caso, las personas reclutadas cuya depresión llevara ocho semanas tendrían un $50 \%$ de probabilidad de remitir espontáneamente en el curso de las ocho semanas habituales que dura un estudio(6).

Los resultados de los ensayos clínicos también se ven afectados por las dificultades para medir la intensidad de la depresión. Habitualmente se emplea la escala de Hamilton, que asigna de 1 a 4 puntos para síntomas como estado de ánimo, sentimientos de culpa, pensamientos suicidas e insomnio. Generalmente, los pacientes son considerados "respondedores" si el puntaje de la escala de Hamilton se reduce al menos en $50 \%$. Pero muchos pacientes en esa situación siguen teniendo importantes síntomas depresivos. Por esta razón, se ha propuesto como criterio alternativo el de "remisión", habitualmente entendido como alcanzar un puntaje menor a 7 puntos en la escala de Hamilton(7). Equivale a la virtual desaparición de la sintomatología depresiva y representa un criterio más restrictivo y difícil de lograr.

Que los enfermos sometidos a un protocolo de investigación mejoren en un alto porcentaje por el solo hecho de participar en él no debiera sorprender. El participante promedio de un estudio de ocho semanas estará siendo entrevistado por un grupo de expertos y evaluadores altamente entrenados alrededor de 20 horas. Por contraste, el paciente depresivo promedio ve al médico unos 20 minutos al mes. Se ha comprobado que el aumento del número de visitas en los estudios farmacológicos se asocia a una reducción paralela de los puntajes de la escala de Hamilton, que es acumulativa y proporcional(8). Se estima que el resultado terapéutico de las evaluaciones de seguimiento puede representar un $40 \%$ del efecto placebo.

Hay factores que predicen una mayor respuesta a placebo en la depresión: la duración breve de la enfermedad, la existencia de un suceso precipitante, la severidad leve o moderada y la buena respuesta previa al tratamiento antidepresivo(9).

En un estudio efectuado en mujeres, se pudo determinar que la respuesta a placebo era mayor en las que tenían un episodio único, en comparación con las que tenían depresión recurrente(10). En las pacientes con un primer episodio, la respuesta a placebo se asoció con menor puntaje inicial de la escala de Hamilton y con menor retardo psicomotor. En las portadoras de depresión recurrente la respuesta a placebo se asoció con menor ansiedad somática.

Respecto de la severidad de la depresión, es más probable la respuesta a placebo en los casos leves (hasta 70\% de mejoría) que en los casos graves (alrededor del 30\% de mejoría)(11,12). Asimismo, la respuesta a placebo depende de la duración del episodio. Si este ha durado más de un año, la respuesta es menor al 30\%; en cambio, si ha durado menos de tres meses se eleva a $50 \%$. Esto puede reflejar la mayor tendencia a la remisión espontánea en las depresiones de corta duración.

Se ha tratado de diferenciar entre el tipo de respuesta verdadera al fármaco y la respuesta a placebo(13). La primera se caracterizaría por un inicio retardado de unas dos semanas y una mejoría persistente. La segunda por una mejoría precoz y transitoria. Es más probable que los pacientes que responden a placebo experimenten más recaídas a pesar del tratamiento de continuación con antidepresivos.

\section{Mecanismos de la respuesta a placebo en depresión}

Varios mecanismos pueden subyacer a la respuesta placebo. Entre ellos se cuentan los factores socioculturales, 
como el sistema de creencias de los pacientes y de los terapeutas, los que pueden diferir del pensamiento y del método científico occidentales. Antropólogos médicos, psiquiatras y psicólogos han estudiado las creencias mágicas, no lógicas, considerándolas aspectos claves del mecanismo del placebo. Cuando un tratamiento carece de una teoría lógica de su acción, la eficacia que se le atribuye deriva de creencias determinadas culturalmente(3).

La respuesta al placebo puede ser determinada de un modo importante por el solo hecho de encontrarse en tratamiento. La incorporación a un protocolo de investigación significa ser sometido a una evaluación completa, tener la oportunidad de hablar sobre el malestar, recibir una explicación del mismo, ser examinado por un terapeuta experto y por un equipo con experiencia y recibir un tratamiento plausible, lo que infunde expectativas de mejoría, entusiasmo y una visión positiva(11). El contacto entre el equipo de investigadores y el paciente puede tener por sí mismo un efecto terapéutico. Por lo tanto, el empleo de placebo no significa que no se esté administrando un tratamiento, sino que se está proporcionando un tratamiento no específico(14). Algunos investigadores han sugerido que las expectativas basadas en el tamaño de las píldoras, su tipo, color y cantidad influyen sobre la mejoría(3). Múltiples cápsulas y de gran tamaño ejercerían un mayor efecto placebo que una sola tableta de tamańo pequeño. Además, el color puede sugerir potencia y eficacia.

La respuesta placebo es fuertemente influida por la calidad de la relación médico-paciente. Una buena relación ayuda a aumentar la adherencia al tratamiento, favorece el efecto placebo y disminuye el efecto nocebo. Pueden jugar un papel fenómenos tales como la transferencia, la sugestión, la reducción de la culpa, la persuasión y el condicionamiento. La convicción que tiene el médico respecto de la potencia del fármaco transmite una poderosa expectativa a un paciente esperanzado y puede constituir un importante mediador de la eficacia terapéutica.

Por eso, en investigación siempre hay que tener presente el "sesgo de la observación o del observador", que consiste en que observadores se sienten inclinados a inducir respuestas por motivos conscientes o inconscientes, o a extraer determinadas conclusiones de los datos(15). Esto lleva a que existan grupos de investigadores más "placebógenos" que otros, lo que puede influir de modo importante en los estudios multicéntricos.

El estudio de los mecanismos neurobiológicos de la respuesta a placebo en depresión es un área de muy reciente desarrollo(16). Los pacientes depresivos que reciben placebo muestran cambios eléctricos y metabólicos en el cerebro. En un estudio se encontró que el placebo inducía cambios electroencefalográficos en la corteza prefrontal, particularmente en el hemisferio derecho(17). Estos hallazgos podrían ser usados para identificar a los sujetos propensos a responder al placebo.

Otro estudio evaluó los cambios en el metabolismo cerebral de la glucosa mediante tomografía por emisión de positrones en pacientes con depresión unipolar(18). El tratamiento con placebo se asoció a aumento del metabolismo en las siguientes regiones: prefrontal, cingulado anterior, premotor, parietal, ínsula posterior y corteza cingulada anterior. Asimismo, hubo disminución del metabolismo en la corteza cingulada subgenual, parahipocampo y tálamo. Es de gran interés que esas regiones también fueron afectadas por fluoxetina (un inhibidor selectivo de la recaptura de serotonina), lo que sugiere un posible rol de la serotonina en el efecto antidepresivo inducido por el placebo(19).

\section{Obligaciones éticas y uso de placebo en estudios con antidepresivos}

Se discute si es éticamente aceptable el empleo de placebo en los estudios clínicos con antidepresivos cuando hay medicamentos eficaces para el tratamiento de la depresión. Existen partidarios de la "ortodoxia del placebo" -quienes consideran que los estudios controlados con placebo son necesarios- y partidarios de la "ortodoxia del control activo" -quienes creen que sólo son legítimos los estudios en los que el nuevo fármaco se compara con un fármaco de eficacia ya establecida(20). Estos últimos sostienen que el empleo de placebo sacrifica la ética, los derechos y el bienestar del paciente en aras de un presunto rigor científico.

La Declaración de Helsinki establece que, en la investigación médica en seres humanos, las consideraciones respecto del bienestar del sujeto tienen precedencia sobre el interés de la ciencia y la sociedad(21). Desde esa perspectiva, los partidarios del uso de control activo sostienen que si existe un tratamiento efectivo debe ser empleado en el grupo control. Según su criterio, lo 
importante no es si un nuevo tratamiento es mejor que nada, sino si es mejor que los tratamientos establecidos. Asimismo, discuten la distinción entre investigación y cuidado clínico(22). No aceptan que, con el argumento de que un ensayo clínico no es una forma de terapia, la ética de la relación médico-paciente no se aplique a la investigación.

Esto es particularmente complejo cuando el investigador tiene una relación terapéutica con el paciente participante del estudio. El médico no debe permitir que su propio interés entre en conflicto con el mejor interés del paciente. El investigador puede estar recibiendo beneficios financieros al reclutar sujetos de investigación o al conducir un ensayo clínico. También puede recibir recompensas profesionales, como publicaciones, promociones o un mayor reconocimiento de sus pares por dirigir la investigación. Esto crea un potencial conflicto de intereses. Los ensayos con una rama placebo no están diseñados para beneficiar al paciente en ese estudio, sino para el beneficio de otros, ya sea futuros pacientes, empresas farmacéuticas, inversionistas u otros. Si la condición de un paciente que está con placebo se deteriora por falta de tratamiento, ello acarrearía cuestionamientos éticos e incluso consecuencias legales. Los argumentos clínicos y científicos para el uso de placebo, cuando hay terapias efectivas establecidas, son poco convincentes y entran en conflicto con reconocidas obligaciones éticas y legales. Los médicos que son investigadores no pueden renunciar a sus obligaciones hacia los pacientes que participan en ensayos clínicos, los que nunca pueden recibir una medicina por debajo de los estándares o quedar en desventaja.

Los partidarios del uso del placebo argumentan que su empleo es ético, incluso en patologías para las cuales hay tratamientos efectivos, por las limitaciones metodológicas de usar un fármaco activo como control. A veces las terapias que se sabe efectivas, en algunos estudios no superan al placebo, por razones tales como las características propias de esa población, las elevadas cifras de mejorías espontáneas, etc. Por lo tanto, si no hay un grupo control, el hallazgo de que no hay diferencias entre el tratamiento establecido y el que se está investigando resulta equívoco o imposible de interpretar(23,24). Si al comparar dos fármacos se encuentran diferencias estadísticamente significativas (e idealmente también clínicamente relevantes) no hay ambigüedad: el nuevo tratamiento es superior o inferior al comparador y debe o no ser adoptado. Pero si no hay diferencias significativas puede haber una o dos razones: ambas son igualmente efectivas o ambas son inefectivas. Esto puede ocurrir aunque el tratamiento establecido haya demostrado eficacia en estudios anteriores. De hecho, un metaanálisis encontró que, en 52 ensayos, sólo el $48 \%$ de los casos con antidepresivos fueron superiores al placebo(25).

Por otra parte, la dicotomía entre ciencia rigurosa y protección ética es falaz. La validez científica constituye una protección ética fundamental(26). La investigación científicamente inválida no puede ser ética, independientemente de la favorable relación entre riesgo y beneficio que tenga para los participantes. En segundo lugar, en algunos casos el riesgo de daño o malestar por el empleo de placebo es inexistente, o tan pequeño que no es razonable establecer como requerimiento ético que el nuevo tratamiento sea probado contra tratamientos establecidos. En tercer lugar, los opositores al placebo no consideran suficientemente su poder, ya que el beneficio terapéutico que proporciona es claramente superior a la ausencia de tratamiento. Por último, los estudios con control activo requieren muestras de pacientes tan altas que muchos de ellos podrían quedar expuestos al riesgo de que el nuevo fármaco sea más ineficaz o tenga más efectos colaterales que el tratamiento establecido. Por ejemplo, si el tratamiento establecido tiene una eficacia del $60 \%$, con un delta de $10 \%$ (en caso de que sean equivalentes, la diferencia entre el tratamiento establecido y el que se investiga deberá ser menor de 10\%), para demostrar equivalencia estadística cada grupo debe contener 279 participantes. En el caso de que se emplee placebo, si la hipótesis es que el placebo tiene una respuesta de $30 \%$ y el tratamiento que se investiga una respuesta de $60 \%$, sólo se requieren 48 participantes en cada grupo. En definitiva, habrá más participantes expuestos a no responder en el grupo en que se compara con un tratamiento establecido que en el grupo en que se compara con placebo(20). En síntesis, los estudios controlados con placebo se justificarían porque sus resultados no son ambiguos, menos sujetos son expuestos a efectos adversos y el número de personas que recibe tratamiento inefectivo es probablemente menor(27).

\section{Alternativas metodológicas al uso de placebo}

La elevada respuesta al placebo en la depresión reduce el poder de los estudios clínicos y dificulta la evaluación de nuevos tratamientos. El desarrollo de nuevos 
antidepresivos se ve complicado por la dificultad de demostrar su superioridad sobre el placebo o su equivalencia al tratamiento establecido. Más de un tercio de los estudios publicados sobre antidepresivos fracasa en demostrar eficacia, por lo que se necesitan nuevas estrategias para reducir sistemáticamente las fuentes de variación(28). Se ha sugerido reclutar sujetos con enfermedad moderada y severa y establecer un período de cuatro semanas en el que reciban educación respecto de cómo manejar su depresión. De este modo, se reduce el número de individuos respondedores a placebo que ingresan en la siguiente etapa del estudio. Otro diseńo consiste en administrar placebo por dos semanas, previo al inicio del estudio comparativo. No obstante, esta estrategia no resulta útil en los estudios en fase aguda(29).

Se han propuesto alternativas al empleo de grupos control que reciben placebo. Entre ellas se encuentran los estudios de adición de fármaco, diseños de dosis variables, establecimiento a priori de un umbral usando un fármaco activo como control para las comparaciones y la comparación con controles históricos(30).

Aunque los estudios con adición de fármacos no eliminan la necesidad de placebo, sí eliminan la monoterapia con placebo. Sin embargo, por la adición del agente primario en los dos grupos en comparación, se requieren grandes poblaciones de sujetos para lograr un poder estadístico suficiente que permita diferenciar entre fármaco y placebo, y también puede influir en la mayor duración del estudio. El empleo de dosis variables del fármaco en estudio permite establecer las dosis apropiadas, pero debe quedar claramente establecido en el consentimiento informado que algunos pacientes recibirán dosis que no son terapéuticas.

El establecimiento de un efecto umbral que el nuevo fármaco debe superar puede obviar la necesidad del control con placebo. No obstante, no se puede excluir la posibilidad de un importante efecto placebo, tanto al establecer el umbral como al administrar el nuevo tratamiento.

La comparación de la eficacia de un nuevo fármaco con los datos históricos de estudios anteriores también se ha sugerido como una alternativa para evitar el empleo de placebo. Sin embargo, la variabilidad de las escalas empleadas y de los criterios diagnósticos y las diferencias temporales en las características clínicas y demográficas de los sujetos limitan su utilidad.

En general, se concuerda en que un estudio bien diseńado puede reducir pero nunca eliminar la respuesta a placebo. El tema continúa siendo un desafío abierto para los investigadores.

\section{Referencias}

1. Kirsch I, Deacon BJ, Huedo-Medina TB, Scoboria A, Moore TJ, Jonson BT. Initial severity and antidepressants benefits: A meta-analysis of data submitted to the Food and Drug Administration. PloS Med 2008; 5: e45.

2. Turner EH, Matthews AM, Linardatos E, Tell RA, Rosenthal R. Selective publication of antidepressant trials and its influence on apparent efficacy. N Engl J Med 2008; 358: 252-260.

3. Straus JL von Ammon, Cavanaught S. Placebo effects. Issues for clinical practice in psychiatry and medicine. Psychosomatics 1996; 37: 315-326.

4. Andrews G. Placebo response in depression: bane of research, boon to therapy (Editorial). Br J Psychiatry 2001; 178: 192-194.

5. Kendler KS, Walters EE, Kessler RC. The prediction of length of major depression episodes: results from an epidemiological survey of female twins. Psychological Medicine 1997; 27: 107-117.

6. Andrews G. Placebo response in depression: bane of research, boon to therapy (Editorial). Br J Psychiatry 2001; 178: 192-194.

7. Thase ME. Remission is the optimal goal of acute phase antidepressant therapy. Actas Esp Psiquiatr 2008; 36(Suppl 1): 7-16

8. Posternak MA, Zimmerman M. Therapeutic effect of follow-up assessments of antidepressants and placebo response rates in antidepressant efficacy trials. Meta-analysis. Br J Psychiatry 2007; 190: 287-292.

9. Brown WA, Khan A. Which depressed patients should receive antidepressants? CNS Drugs 1994; 5: 341-347.

10. Bialik RJ, Ravindan AV, Bakish D, Lapierre YD. A comparison of placebo responders and nonresponders in subgroups of depressive disorders. J Psychiatry Neurosci 1995; 20: 265-270. 
El efecto placebo en los ensayos clínicos con antidepresivos - Hernán Silva Ibarra

11. Khan A, Brown WA. The placebo enigma in antidepressant clinical trials. J Clin Psychopharmacol 2001; 21: 123-125.

12. Sonawalla SB, Fava M. Severe depression: is there a best approach? CNS Drugs 2001; 15: 765-776.

13. Stewart JW, Quitkin FM, McGrath PJ, et al. Use of pattern analysis to predict differential relapse of remitted patients with major depression during 1 year of treatment with fluoxetine or placebo. Arch Gen Psychiatry 1998; 55: 334-343.

14. Ranga RK. Efficient trial design to reduces placebo requirement. Biol Psychiatry 2000; 47: 724-726.

15. Lolas F. Depresión y efecto placebo: un tema de investigación bioética. Monografias de Psiquiatría 2003; 15(3): 17 20.

16. Price DD, Finniss DG, Benedetti F. A comprehensive review of the placebo effect: recent advances and current thought. Annu Rev Psychol 2008; 59: 565-590.

17. Leuchter AF, Cook IA, Witte EA, Morgan M, Abrams M. Changes in brain function of depressed subjects during treatment with placebo. Am J Psychiatry 2002; 159: 122-129.

18. Mayberg HS, Silva JA, Brannan SK, Tekell JL, Mahurin RK, McGinnis S, Jerabek PA. The functional neuroanatomy of the placebo effect. Am J Psychiatry 2002; 159: 728-737.

19. Benedetti F, Mayberg HS, Wager TD, Stholer CS, Zubieta JK. Neurobiological Mechanisms of the placebo effect. J Neurosci 2005; 25: 10390-10402.

20. Ezekiel JE, Miller FG. The ethics of placebo-controlled trials. A middle ground. $N$ England J Medicine 2001; 345: 915-919.

21. World Medical Assembly. Declaration of Helsinki. Geneva, Switzerland: World Health Organization; 1964.

22. Glass KC. Ethical obligations and the use of placebo controls. Can J Psychiatry 2008; 53: 428-429.

23. Temple R, Ellenberg SS. Placebo-controlled trials and active-control trials in the evaluation of new treatments. I. Ethical and scientific issues. Ann Intern Med 2000; 133: 455-463.

24. Ellenberg SS, Temple R. Placebo-controlled trials and active-control trials in the evaluation of new treatments. II. Practical issues and specific cases. Ann Intern Med 2000; 133: 464-470.

25. Kahn A, Kahn S, Brown WA. Are placebo controls necessary to test new antidepressants and anxiolytics? Int J Neuropsychopharmacol 2002; 5: 193-197.

26. Emanuel EJ, Wendler D, Grady C. What makes clinical research ethical? JAMA 2000; 283: 2701-2711.

27. Streiner DL. The lesser of 2 evils: the ethics of placebo-controlled trials. Can J Psychiatry 2008; 53(7): 430-432.

28. Thase ME. How should efficacy be evaluated in randomized clinicals trials of treatment for depression? J Clin Psychiatry 1999; 60(Suppl 4): 23-31.

29. Trivedi MH, Rush H. Does a placebo run-in or a placebo treatment cell affect the efficacy of antidepressants medications? Neuropsychopharmacology 1994; 11: 33-43.

30. Keck PE, Welge JA, McElroy SL, Arnold LM, Strakowski LM. Placebo effect in randomized, controlled studies of acute bipolar mania and depression. Biol Psychiatry 2000; 47: 748-755.

Recibido: 6 de octubre de 2008

Aceptado: 28 de octubre de 2008 